\title{
Coulomb interactions at quantum Hall critical points of systems in a periodic potential
}

\author{
Jinwu Ye \\ Department of Physics, The Johns Hopkins University, Baltimore MD, 21218 \\ Subir Sachdev \\ Department of Physics, P.O. Box 208120, Yale University, New Haven, CT 06520-8120
}

(August 1, 2018)

\begin{abstract}
We study the consequences of long-range Coulomb interactions at the critical points between integer/fractional quantum Hall states and an insulator. We use low energy theories for such transitions in anyon gases in the presence of an external periodic potential. We find that Coulomb interactions are marginally irrelevant for the integer quantum Hall case. For the fractional case, depending upon the anyon statistics parameter, we find behavior similar to the integer case, or flow to a novel line of fixed points with exponents $z=1$, $\nu>1$ stable against weak disorder in the position of the critical point, or runaway flow to strong coupling.
\end{abstract}

Typeset using REVTEX 
The zero temperature quantum phase transitions between the different quantum Hall and insulating states of a two-dimensional electron gas in a strong magnetic field are among the most intensively studied quantum critical points, both theoretically [1, 2] and experimentally [3]. Earlier theoretical investigations focussed on the transitions between the integer quantum Hall plateaus and described them in terms of non-interacting electrons moving in a random external potential [4]. It has also been argued that the transitions between fractional quantum Hall states could be mapped onto models essentially equivalent to those between the integer states [5]. The latter point of view was however questioned by Wen and $\mathrm{Wu}$ [6] and Chen, Fisher and Wu [7]: they focussed on the simpler case of systems in the presence of a periodic rather than a random potential, and examined a model of anyons, with a statistical angle $\theta$ and short-range repulsive interactions, which displayed a second order quantum phase transition between a quantized Hall state and a Mott insulator as the strength of the periodic potential was varied. This transition was characterized by a line of critical points with continuously varying exponents, parametrized by the value of $\theta$. For the case $\theta=0$, when the anyons were fermions, the transition was out of a integer quantum Hall state; its exponents and other universal properties were different from the cases $0<\theta<2 \pi$ for which the anyons acquired fractional statistics and the transition was out a fractional quantum Hall state. (For $\theta=2 \pi$ the anyons became bosons and the Hall state reduced to a superfluid.)

In all of the above theoretical works, the long-range Coulomb interactions between charge carriers have been effectively ignored. However, a few recent works have taken steps to remedy this serious shortcoming. Yang et al. [8] studied the integer quantum Hall transition under a Hatree-Fock treatment of the Coulomb interaction. Lee and Wang [9] showed that the renormalization group eigenvalue of the Coulomb interaction was zero at the Hartree-Fock critical point; higher order calculations are therefore necessary to understand the physics. Pfannkuche and MacDonald [10] numerically studied electrons with Coulomb interactions in a periodic potential between a fractional Hall state and an insulator, but were limited to rather small system sizes. Interesting scaling interpretations of Coulomb interaction-induced 
dephasing were discussed in Ref [1].

In this paper we shall provide a thorough analysis of the consquences of Coulomb interactions on the anyons in a periodic potential model of Refs [6,77. We shall show that the Coulomb interaction is marginally irrelevant for the integer case $(\theta=0)$, and remains so for the fractional case for small values of $\theta$; this marginally irrelevant interaction will lead to logarithmic corrections to naive scaling functions for the vicinity of the transition. For larger $\theta$, we will establish, in a certain $1 / N$ expansion, the existence of a novel line of fixed points at which the Coulomb interactions acquire a non-zero fixed point value determined by the value of $\theta$. There are no logarithmic corrections at these fixed points, and naive scaling holds. We find a dynamic critical exponent $z=1$ at all points on the fixed line, providing a concrete realization of the scenario [12,2], not previously established explicitly, that energies must scale as inverse distances for the $1 / r$ Coulomb interaction. It is also worth noting that, despite the value $z=1$, the critical correlators are not Lorentz invariant. We also find that the correlation length exponent $\nu$ satisfies $\nu>2 / d$ (where $d=2$ is the spatial dimensionality) along this fixed line, which implies that the fixed line is stable towards disorder involving local fluctuations in the position of the critical point.

We begin our analysis by writing down the model of Ref [7] extended to include Coulomb interactions between the charge carriers

$$
\begin{array}{rl}
\mathcal{L}=\int d^{d} & x d \tau\left[\alpha \bar{\psi}_{m} \gamma_{0} \partial_{0} \psi_{m}+\bar{\psi}_{m} \gamma_{i} \partial_{i} \psi_{m}\right. \\
& \left.-\frac{i}{\sqrt{N}} q \mu^{\epsilon / 2} \alpha^{1 / 2} a_{0} \bar{\psi}_{m} \gamma_{0} \psi_{m}-\frac{i}{\sqrt{N}} g \mu^{\epsilon / 2} \alpha^{1 / 2} a_{i} \bar{\psi}_{m} \gamma_{i} \psi_{m}\right] \\
& +\int \frac{d^{2} k}{4 \pi^{2}} \frac{d \omega}{2 \pi}\left[i k a_{0}(-\vec{k},-\omega) a_{t}(\vec{k}, \omega)+\frac{k}{2} a_{t}(-\vec{k},-\omega) a_{t}(\vec{k}, \omega)\right]
\end{array}
$$

The $\psi_{m}$ are $m=1 \cdots N$ species of charge $q / \sqrt{N} 2+1$ dimensional Dirac fermions which interact with a $U(1)$ gauge field $\left(a_{0}, a_{i}\right)(i=1,2)$; we are interested in the case $N=1$ but will find the large $N$ expansion to be a useful tool. The $\gamma_{0}, \gamma_{i}$ are the Dirac $\gamma$ matrices, $x_{i}(\tau)$ are spatial (temporal) co-ordinates with $\partial_{0} \equiv \partial_{\tau}, \partial_{i} \equiv \partial_{x_{i}}$, and $\vec{k}, \omega(k=|\vec{k}|)$ are the Fourier transformed wavevector and frequency variables. To aid the subsequent renormalization 
group analysis, we are working in $d=2+\epsilon$ spatial dimensions and $\mu$ is a renormalization scale. The parameter $\alpha$ is introduced to allow for anisotropic renormalization between space and time [13]. We have used the Coulomb gauge which allows us to explicitly represent $a_{i}$ in terms of the transverse spatial component with $a_{i}=i \epsilon_{i j} k_{j} a_{t} / k$. The term before last in $\mathcal{L}$ is the Chern Simons coupling: it turns the Dirac particles into anyons with a statistical angle $\theta / N$ with $\theta \equiv q g$; notice that the angle is of order $1 / N$ and so the expected periodicity of the physics under $\theta / N \rightarrow \theta / N+4 \pi$ will not be visible in the $1 / N$ expansion. The last term is the Coulomb interaction, and it has been written in terms of $a_{t}$ following Ref [14.

In the absence of the last Coulomb interaction term, it was shown in Ref $[7]$ that $\mathcal{L}$ represents the critical theory of a system of anyons in a periodic potential undergoing a transition from an insulator with conductivities $\sigma_{x x}=\sigma_{x y}=0$ into a fractional quantum Hall state with $\sigma_{x x}=0$ and $\sigma_{x y}=\left(q^{2} / h\right) /(1-\theta / 2 \pi)$ to leading order in $1 / N$. Both these states have energy gaps, and we have shown that the Coulomb interaction does not modify the values of $\sigma_{i j}$ in either phase. The relationship of the continuum model $\mathcal{L}$ to the more realistic model of electrons studied in Ref [10] remains somewhat unclear, although it is plausible that $\mathcal{L}$ is the critical theory of the latter. We may also view $\mathcal{L}$ as the simplest theory consistent with the following requirements, and therefore worthy of further study: ( $i$ ) the two phases on either side of the critical point have the correct values of $\sigma_{i j}$, and the Hall phase has both quasi-particle and quasi-hole excitations with the correct charge and statistics, and ( $i i)$ the gap towards the quasi-particle and the quasi-hole excitations vanishes at the critical point.

We now proceed with a renormalization group analysis of $\mathcal{L}$. Simple power counting shows that both the Chern-Simons and Coulomb interactions [15] are marginal at tree level in $d=2$, and so loop expansions are required and useful. Power counting also shows that a short-range four-fermion interaction term is irrelevant and has therefore been neglected in $\mathcal{L}$; this makes the fermionic formulation of the anyon problem much simpler than its bosonic counterpart [6, 16, 117].

The loop expansion requires counterterms to account for ultraviolet divergences in mo- 
mentum integrals; we write the counter terms as

$$
\begin{aligned}
\mathcal{L}=\int d^{2} x d \tau\left[\alpha\left(Z_{\alpha}-1\right) \bar{\psi}_{m} \gamma_{0} \partial_{0} \psi_{m}+\left(Z_{2}-1\right) \bar{\psi}_{m} \gamma_{i} \partial_{i} \psi_{m}\right. \\
\left.-\frac{i}{\sqrt{N}}\left(Z_{1}^{q}-1\right) q \mu^{\epsilon / 2} \alpha^{1 / 2} a_{0} \bar{\psi}_{m} \gamma_{0} \psi_{m}-\frac{i}{\sqrt{N}}\left(Z_{1}^{g}-1\right) g \mu^{\epsilon / 2} \alpha^{1 / 2} a_{i} \bar{\psi}_{m} \gamma_{i} \psi_{m}\right]
\end{aligned}
$$

In general, counter terms for the last two gauge field terms in $\mathcal{L}$ should also be considered. However, we have shown [17 that at least to two loops, there no divergences associated with these terms. The Ward identities following from gauge invariance dictate $Z_{1}^{q}=Z_{\alpha}$ and $Z_{1}^{g}=Z_{2}$. Using these identities we relate the bare fields and couplings in $\mathcal{L}$ to the renormalized quantities by $\psi_{m B}=Z_{2}^{1 / 2} \psi_{m}, \alpha_{B}=\left(Z_{\alpha} / Z_{2}\right) \alpha, q_{B}=q \mu^{\epsilon}\left(Z_{\alpha} / Z_{2}\right)^{1 / 2}$ and $g_{B}=$ $g \mu^{\epsilon / 2}\left(Z_{2} / Z_{\alpha}\right)^{1 / 2}$. Notice that these relations imply that for the statistical angle $\theta / N=q g / N$ we have $\theta_{B}=\theta \mu^{\epsilon}$; so in $d=2$ this angle is a renormalization group invariant, which is expected on general physical grounds. The dynamic critical exponent, $z$ is related to the renormalization of $\alpha$ by 13

$$
z=1-\mu \frac{d}{d \mu} \ln \alpha=1-\mu \frac{d}{d \mu} \ln \frac{Z_{2}}{Z_{\alpha}}
$$

We will find it convenient to express the loop expansion in terms of the "fine structure" constant $w \equiv \pi q^{2} / 8$, and a central object of study shall be its $\beta$-function $\beta(w)=\mu(d w / d \mu)$. By comparing (3) with relationships between bare and renormalized quantities quoted above we see that

$$
z=1-\beta(w) / w
$$

Finally, the critical exponent $\nu$ is related to the anomalous dimension of the composite operator $\bar{\psi} \psi$ by $\nu^{-1}-1=\mu\left(d \ln Z_{\bar{\psi} \psi} / d \mu\right)$; the renormalization constant $Z_{\bar{\psi} \psi}$ can be calculated by inserting the operator into the self-energy diagrams.

We begin the explicit calculation of the renormalization constants by considering a direct perturbative expansion in the Coulomb fine structure constant $w$ and the statistical andgle $\theta$. At one-loop order, we find no dependence on $\theta$; the values of the renormalization constants upto terms of order $w^{2}, \theta^{2}$ and $w \theta$ are 


$$
Z_{2}=1-2 w / N \pi \epsilon, \quad Z_{\alpha}=1, \quad Z_{\bar{\psi} \psi}=Z_{2}
$$

We also explicitly verified that the gauge invariance Ward identities hold. From these results we find for the $\beta$-function of the Coulomb coupling

$$
\beta(w)=\frac{2 w^{2}}{N \pi}+\mathcal{O}\left(w^{3}, w^{2} \theta^{2}\right)
$$

while the critical exponents are

$$
z=1-2 w / N \pi, \quad \nu=1-2 w / N \pi
$$

to be evaluated at the fixed point of the $\beta$-function. The result (6) shows that the $w$ is marginally irrelevant and flows to the fixed point $w^{*}=0$ at long distances. During this flow (7) shows that the effective $z<1$, corresponding to a smaller density of states at low energies, which is physically consistent with the irrelevance of Coulomb interactions. For the integer Hall state case we have $\theta=0$, and then the fixed point is simply a free Dirac theory: in this case the Coulomb interactions are dangerously irrelevant, as it is responsible for the $T$ dependence of physical quantities and will lead to logarithmic corrections to naive scaling.

To understand larger values of $\theta$, and to explore the consequences of a possible interference between the Coulomb interactions and the Chern-Simons term we found it convenient to perform a $1 / N$ expansion. This is technically simpler than a perturbative two-loop extension of the computation above, and also automatically includes the dynamic screening of the gauge field propagator by the fermion polarization [14]. Alternatively stated, the so-called RPA approximation becomes exact at $N=\infty$, and $1 / N$ corrections require gauge field propagators which have the RPA form

$$
\mathcal{L}_{R P A}=\frac{1}{2} \int \frac{d^{2} k}{4 \pi^{2}} \frac{d \omega}{2 \pi}\left(a_{0}, a_{t}\right)\left(\begin{array}{cc}
q^{2} k^{2} /\left(16 \sqrt{k^{2}+\omega^{2}}\right) & i k \\
i k & k+g^{2} /\left(16 \sqrt{k^{2}+\omega^{2}}\right)
\end{array}\right)\left(\begin{array}{c}
a_{0} \\
a_{t}
\end{array}\right)
$$

Evaulating the fermion self-energy diagrams to order $1 / N$, we find for the renormalization constants 


$$
\begin{aligned}
& Z_{2}=1-\frac{1}{N \epsilon}\left(\frac{2 w}{\pi \lambda}-\frac{16 w^{2} A}{\pi^{2} \lambda}+\frac{\theta^{2} C}{16 \pi^{2}}-\frac{\theta^{2} E}{16 \pi^{2}}\right) \\
& Z_{\alpha}=1-\frac{1}{N \epsilon}\left(\frac{16 w^{2} B}{\pi^{2} \lambda}-\frac{\theta^{2} D}{16 \pi^{2}}+\frac{\theta^{2} F}{16 \pi^{2}}\right)
\end{aligned}
$$

where $\lambda=1+(\theta / 16)^{2}$ and the constants $A, B, C, D, E=A+B, F=B$ are given by the formal expressions

$$
\begin{aligned}
& A=\int_{0}^{1} d x \frac{4 x^{2}\left(1-x^{2}\right)}{\left(1+x^{2}\right)^{3}} f(x ; w, \theta), \quad B=\int_{0}^{1} d x \frac{\left(1-x^{2}\right)\left(1-6 x^{2}+x^{4}\right)}{\left(1+x^{2}\right)^{3}} f(x ; w, \theta) \\
& C=\int_{0}^{1} d x \frac{4 x^{2}}{\left(1-x^{2}\right)\left(1+x^{2}\right)} f(x ; w, \theta), \quad D=\int_{0}^{1} d x \frac{\left(1-6 x^{2}+x^{4}\right)}{\left(1-x^{2}\right)\left(1+x^{2}\right)} f(x ; w, \theta)
\end{aligned}
$$

with $f(x ; w, \theta)=\left(\lambda\left(1+x^{2}\right)+w\left(1-x^{2}\right)\right)^{-1}$, and the variable $x$ represents an intermediate frequency. Note the two constants $C, D$ are divergent: this divergence is due to the singular effect of frequencies $|\omega| \gg k$. However, as shown below and in Ref [17], these divergences are gauge artifacts and cancel in the $\beta$-function and in any physical gauge-invariant quantity like $\nu, z$ or $\sigma_{i j}$. The divergences however do infect the anomalous dimension of the field operator $\psi$ : this is as expected as the propagator of $\psi$ is clearly gauge-dependent.

We computed the $\beta$-function of the Coulomb coupling $w$ from the renormalization constants (9); the divergences do indeed cancel and we obtain the result

$$
\begin{aligned}
\beta(w) & =\frac{2 w^{2}(1-\phi)}{N \pi^{2} \lambda^{2}}\left[\pi-16 w \int_{0}^{1} d x\left(\frac{1-x^{2}}{1+x^{2}}\right)^{3} \frac{\lambda\left(1+x^{2}\right)+\frac{w}{2}\left(1-x^{2}\right)}{\left(\lambda\left(1+x^{2}\right)+w\left(1-x^{2}\right)\right)^{2}}\right] \\
& +\frac{32 w \phi}{N \pi^{2}} \int_{0}^{1} d x \frac{\left(1-x^{2}\right)\left(-1+10 x^{2}-x^{4}\right)}{\left(1+x^{2}\right)^{3}} \frac{\left(1+x^{2}\right)+\frac{w}{2}\left(1-x^{2}\right)}{\left(\lambda\left(1+x^{2}\right)+w\left(1-x^{2}\right)\right)^{2}},
\end{aligned}
$$

where $\phi \equiv(\theta / 16)^{2}$ and $\lambda=1+\phi$. In a similar manner, the effective exponent $z$ is given by (舟), and for the exponent $\nu$ we obtain

$$
\begin{aligned}
& \frac{1}{\nu}=1- \frac{128 \phi}{N \pi^{2}} \int_{0}^{1} d x \frac{\left(1-x^{2}\right)\left(1+6 x^{2}+x^{4}\right)}{\left(1+x^{2}\right)^{3}} \frac{1+x^{2}+\frac{w}{2}\left(1-x^{2}\right)}{\left(\lambda\left(1+x^{2}\right)+w\left(1-x^{2}\right)\right)^{2}} \\
&+\frac{512 \phi(1-\phi)}{N \pi^{2}} \int_{0}^{1} d x \frac{\left(1-x^{2}\right)\left(1+x^{2}\right)}{\left(\lambda\left(1+x^{2}\right)+w\left(1-x^{2}\right)\right)^{3}}
\end{aligned}
$$

in this last expression we have used the fact that $\beta(w)=0$ at a fixed point to simplify the result a bit. In the absence of Coulomb interactions $(w=0)$ the above result for $\nu$ becomes: 


$$
\nu=1-\frac{512 \phi(1-2 \phi)}{N 3 \pi^{2} \lambda^{3}}
$$

It agrees with earlier results [7, 18] obtained in a very different computation in the Lorentz gauge: this agreement is another non-trivial check of our renormalization procedure.

We now turn to the physical implications of our main results (11) and (12).

First, consider the transition out of the integer quantum Hall state, $\theta=0$, which implies $\phi=0, \lambda=1$. Then simple analysis of (11) shows that $\beta(w)>0$ for all $w>0$; for small $w$ we have $\beta(w)=2 w^{2} / N \pi$, in agreement with one-loop result (6), while for $w \gg 1$, $\beta(w)=4 / N \pi$. So the only fixed point remains at $w=0$, and the prediction of the large $N$ theory agrees with the weak-coupling analysis-Coulomb interactions are dangerously irrelevant. This agreement between the two approaches is reassuring as it is not a priori required: it is absent in the bosonic formulation [6] of the same critical point.

Turning to the fractional case with a non-zero $\theta$, we show a plot of a numerical integration of the flows implied by (11) in Fig 1; there are three distinct regimes:

(i) $\phi<\phi_{c 1}$

For small values of $\phi$ the $w=0$ fixed point remains stable, as for the integer case above. The limiting value $\phi_{c 1}$ can be determined by expanding $\beta(w)$ in (11) in powers of $w$ :

$$
\beta(w)=\frac{w^{2}}{2 N \pi \lambda^{3}}\left(4+3 \phi-5 \phi^{2}\right)-\frac{32 w^{3}}{15 N \pi^{3} \lambda^{4}}\left(5+4 \phi-7 \phi^{2}\right)
$$

The co-efficient of $w^{2}$ changes sign at $\phi=\phi_{c 1}=(3+\sqrt{89}) / 10 \approx 1.24$, beyond which the $w=0$ point is no longer stable.

(ii) $\phi_{c 1}<\phi<\phi_{c 2}$

For intermediate values of $\phi$, the flow is towards an attractive line of fixed points $0<$ $w^{*}(\phi)<\infty$. The value $\phi=\phi_{c 2}$ at which $w^{*}(\phi) \rightarrow \infty$ can be determined by evaluating (11) in the large $w$ limit:

$$
\beta(w \rightarrow \infty)=\frac{2(2-\phi)}{N \pi}
$$

This shows that the flow is away from $w=\infty$ for $\phi<\phi_{c 2}=2$. This line of stable fixed points for the present range of $\phi$ is our main new result. We can easily determine the values 
of the fixed-point critical exponents: from (4) we see that the $z=1$, while $\nu$ follows from (12). We find $\nu\left(\phi_{c 1}\right) \approx 1+2.82 / N$, and $\nu\left(\phi_{c 2}^{-}\right)=1+1 / N w^{*}$, and a monotonic change in between. As noted earlier, because $\nu>1$, this line is stable to disorder in the local position of the critical point.

(iii) $\phi>\phi_{c 2}$

Now the flow is to $w^{*}=\infty$. However, the flows cannot be trusted once $w \sim N$, and so we are unable to draw any firm conclusions about this regime.

To conclude, we have presented an analysis of the consequences of Coulomb interactions at quantum Hall critical points which goes well beyond the linear stability/Hartree-Fock treatments in earlier works [8,9]. To allow such a study, we simplified the usual physical situation by replacing the random external potential by a periodic one. Nevertheless, it is quite interesting that we found a fixed line which is stable towards the introduction of small disorder in the position of the critical point.

We thank M.P.A. Fisher, B. Halperin, M. Franz, C. Kane, S. Kivelson, A. Millis, N. Read, R. Shankar, Z. Wang and Y. S. Wu for helpful discussions. This work was initiated at the Aspen Center for Physics and supported by NSF Grant No. DMR-97-07701 (J.Ye) and DMR-96-23181 (S.S.) 


\section{REFERENCES}

[1] B. Huckenstein, Rev. Mod. Phys. 67, 357 (1995).

[2] S.L. Sondhi, S.M. Girvin, J.P. Carini and D. Shahar, Rev. Mod. Phys. 69, 315 (1997).

[3] L.W. Engel, D. Shahar, C. Kurdak, and D.C. Tsui, Phys. Rev. Lett. 71, 2638 (1993); H.P. Wei, L.W. Engel and D.C. Tsui, Phys. Rev. B 50, 14609 (1994); D. Shahar, D.C. Tsui, M. Shayegan, E. Shimshoni and S.L. Sondhi, Science, 274, 589 (1996).

[4] A.M.M. Pruisken in The Quantum Hall Effect, R.E. Prange and S.M. Girvin eds, Springer-Verlag, New York (1990); J.T. Chalker and P.D. Coddington, J. Phys. C 21, 2665 (1988).

[5] J.K. Jain, S. Kivelson and N. Trivedi, Phys. Rev. Lett. 64, 1297 (1990); S. Kivelson, D.H. Lee and S.C. Zhang, Phys. Rev. B 46, 2223 (1992).

[6] X.G. Wen and Y.S. Wu, Phys. Rev. Lett. 70, 1501 (1993).

[7] W. Chen, M.P.A. Fisher and Y.S. Wu, Phys. Rev. B 48, 13749, (1993).

[8] S.R. Eric Yang and A.H. MacDonald, Phys. Rev. Lett. 70, 4110 (1993); S.R. Eric Yang, A.H. MacDonald and B. Huckenstein, ibid 74, 3229 (1995).

[9] D.-H. Lee and Z. Wang, Phys. Rev. Lett. 76, 4014 (1996).

[10] D. Pfannkuche and A.H. MacDonald, Phys. Rev. B 56, R7100 (1997) .

[11] D.G. Polyakov and K.V. Samokhin, report No. cond-mat/9705154.

[12] M.P.A. Fisher, Phys. Rev. Lett. 65, 923 (1990).

[13] E. Brezin and J. Zinn-Justin, Phys. Rev. B 13, 251 (1976); D. Boyanovsky and J. Cardy, ibid, 26, 154 (1982).

[14] B.I. Halperin, P.A. Lee and N. Read, Phys. Rev. B 47, 7312 (1993).

[15] S. Sachdev, report No. cond-mat/9709243. 
[16] M.P.A. Fisher and G. Grinstein, Phys. Rev. Lett. 60, 208 (1988).

[17] J. Ye and S. Sachdev, unpublished.

[18] The agreement appears after correction of a technical error in Ref. [7]. 


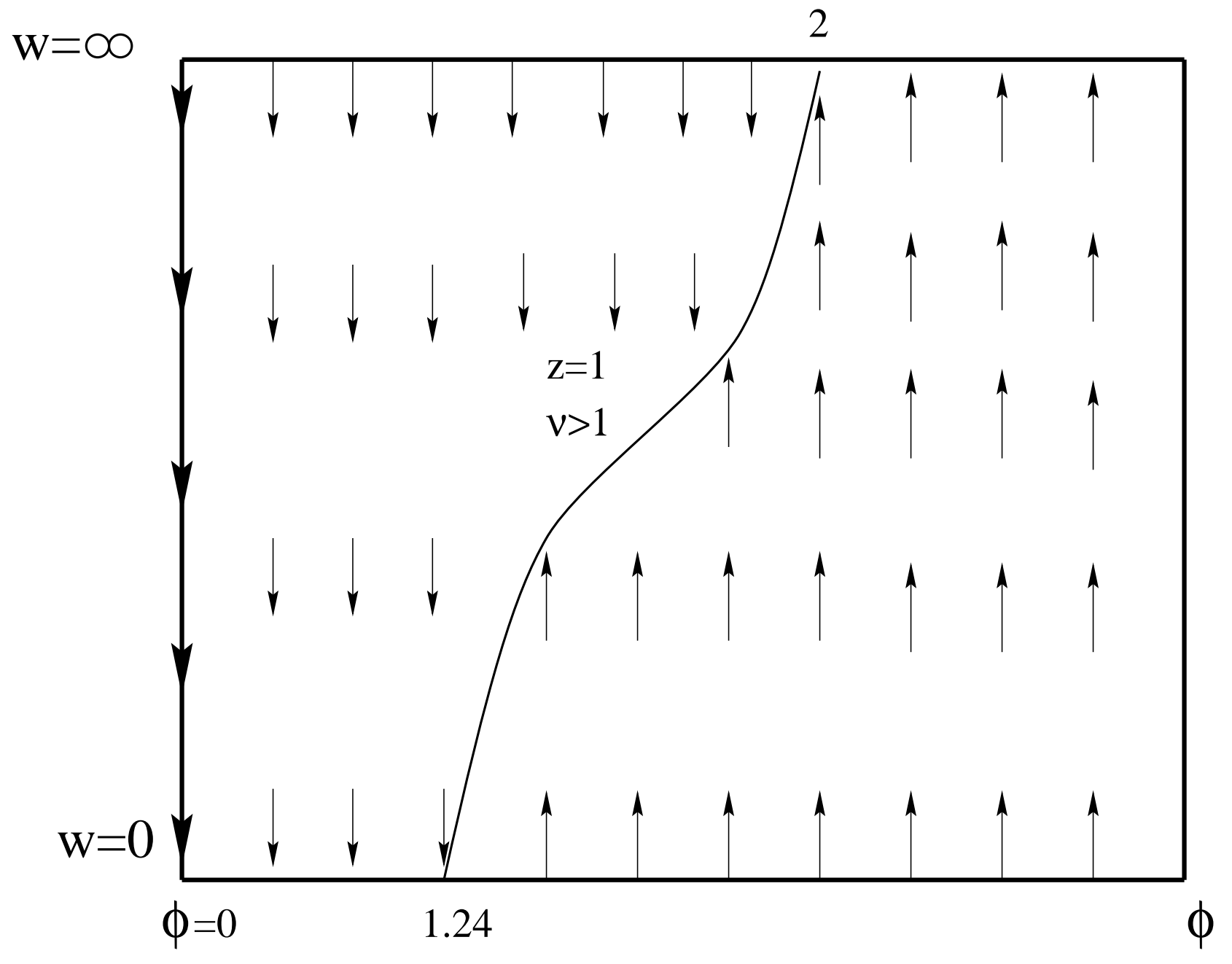

Fig.1 R. G. flow of Coulomb and Chern-Simon couplings 